\title{
Genomic insertion locus and Cas9 expression in the germline affect CRISPR/Cas9-based gene drive performance in the yellow fever mosquito Aedes aegypti
}

William R Reid ${ }^{1}$, Jingyi Lin ${ }^{1}$, Adeline E Williams ${ }^{2}$, Rucsanda Juncu ${ }^{1}$, Ken E Olson ${ }^{2}$, Alexander WE Franz ${ }^{1 *}$

${ }^{1}$ Department of Veterinary Pathobiology, University of Missouri, Columbia, MO 65211, USA.

${ }^{2}$ Department of Microbiology, Immunology, and Pathology, Colorado State University, Fort Collins, CO 80523, USA.

${ }^{*}$ Corresponding author franza@missouri.edu

\begin{abstract}
The yellow fever mosquito Aedes aegypti is a major vector of arthropod-borne viruses, including dengue, chikungunya, and Zika. A novel approach to mitigate arboviral infections is to generate mosquitoes refractory to infection by overexpressing antiviral effector molecules. Such an approach requires a mechanism to spread these antiviral effectors through a population, for example, by using CRISPR/Cas9-based gene drive systems. Here we report an autonomous single-component gene drive system in Ae. aegypti that is designed for persistent population replacement. Critical to the design of a single-locus autonomous gene drive is that the selected genomic locus be amenable to both gene drive and the appropriate expression of the antiviral effector. In our study, we took a reverse engineering approach to target two genomic loci ideal for the expression of antiviral effectors and further investigated the use of three promoters for Cas9 expression (nanos, 62-tubulin, or zpg) for the gene drive. We found that both promoter selection and genomic target site strongly influenced the efficiency of the drive, resulting in $100 \%$ inheritance in some crosses. We also observed the formation of inheritable gene drive blocking indels (GDBI) in the genomic locus with the highest levels of gene drive. Overall, our drive system forms a platform for the further testing of driving antipathogen effector genes through Ae. aegypti populations.
\end{abstract}




\section{Significance statement}

CRISPR/Cas9 based gene drives for the purposes of population replacement have been developed in Anopheles spp. and self-exhausting split drives have been developed in Ae. aegypti. While these studies show a high level of gene drive, they are exclusively targeted to eye pigmentation genes, which restricts the available number of genomic loci for integration. Given that the genomic locus strongly influences the expression of transgenes, the goal of our work was to migrate an autonomous CRISPR/Cas9 gene drive system into genomic loci known to allow for strong antiviral gene expression in Ae. aegypti, allowing the testing of CRISPR/Cas9 based gene drives to be independent of eye marker genes.

\section{Introduction}

The yellow fever mosquito Aedes aegypti is the principal vector for arthropod-borne viruses (arboviruses) such as dengue, yellow fever, chikungunya, and Zika in tropical regions of the world (Weaver and Vasilakis, 2009; Weaver and Reisen, 2010; Liu-Helmersson et al., 2019). Presently, the major strategy to control Ae. aegypti populations relies on the use of Bacillus thuringiensis var israelensis (Bti) and chemical insecticides (FKMCD 2019; Manjarres-Suarez and Olivero-Verbel, 2013); however, these approaches have led to multiple mechanisms of insecticide resistance, warranting the ongoing quest for alternative methods of control (Martins et al., 2009; Manjarres-Suarez and OliveroVerbel, 2013; Liu 2015; Du et al., 2016; Saavedra-Rodriguez et al., 2018; Fan and Scott, 2020; Schluep and Buckner, 2021). Novel approaches, including the incompatible insect technique (IIT) using the intracellular parasite Wolbachia (Kittayapong et al. 2019; Beebe et al., 2021) and transgenic genetic suppression techniques mosquitoes with a dominant lethal transgene (RIDL, fsRIDL), have been tested in the field (Labbé et al., 2012; Waltz 2021). Other promising technologies, including precision-guided sterile insect technique (pgSIT) have been developed for the purposes of localized, confinable management of Ae. aegypti populations (Li et al., 2020; Li et al., 2021). Another genetic control strategy, termed population replacement, aims at spreading an antipathogen (i.e., antiviral) effector through a targeted mosquito population. Antiviral effectors, specifically blocking dengue and Zika viruses in Ae. aegypti, have been previously developed and tested (Franz et al., 2006, 2014; Yen et al., 2018; Buchman et al., 2019, 2020; Williams et al., 2020). Linking a powerful (autonomous) gene drive system to the antiviral effector could lead to super-Mendelian inheritance of the transgene within the targeted mosquito population, thereby overcoming potential fitness effects associated with the antiviral effector (Irvin et al., 2004; Moreira et al., 2004). As a consequence, the proportion of the population harboring the effectors would be increased, resulting in the emergence of mosquitoes refractory to arbovirus 
transmission. For such a broad-scale population replacement approach, the gene drive system needs to be highly invasive. Recently, highly invasive autonomous gene drives that require low release thresholds and reach fixation within a few generations in cage trials have been developed for Anopheles spp. (Gantz et al., 2015; Carballar-Lejarazú et al., 2020; Adolfi et al., 2020). Gene drive systems utilizing a homing endonuclease gene (HEG) based approach were initially proposed in 2003 and subsequently demonstrated in 2008 in An. gambiae as a fully synthetic homing drive using the DNA cleaving intronencoded endonulease Ppol-I (Burt, 2003; Windbichler et al., 2007). Recently, the CRISPR/Cas9 system has been modified to allow for HEG-based drives in Drosophila melanogaster (Gantz and Bier, 2015), along with several mosquito species including Anopheles stephensi (Gantz et al., 2015; Adolfi et al., 2020), An. gambiae (Carballar-Lejarazú et al., 2020), and Ae. aegypti (Li et al., 2020; Verkuijl et al., 2020). The HEG drive developed in Ae. aegypti inserted itself into the white gene on chromosome 1 and contained a single guide RNA (sgRNA)-expressing cassette that targeted the locus, along with an eGFP eye marker as cargo. Initially, highly invasive gene drive systems were thought to carry substantial environmental risk since they are not designed to self-eliminate or be confinable to a region. However, several systems have been developed that would allow for their recall, including systems that destroy or overwrite the drive, and more recently, a "biodegradable" gene drive system (Wu et al. 2016; Gantz and Bier 2016; Chae et al. 2020; Zapletal et al., 2020). For local, temporary releases, split-drive gene drive systems have been developed for Ae. aegypti, which - by design - are modelled to be self-limiting or self-extinguishing (Li et al., 2020; Verkuijl et al., 2021). In these split drive systems, the activity of Cas9expressing lines was leveraged while Cas9 expression was driven by different promoters, including exuperantia, 4nitro, trunk, nup50, and PUb (Li et al., 2017, Li et al., 2020). The work by Li et al (2020) demonstrated an efficient gene drive system that was designed to self-eliminate over time and, thus, be confinable. While the capacity of a gene drive to be inherently self-eliminating mitigates the risk of negative ecological consequences, it also limits the scale of the release and requires a higher release threshold to cause an effect in the field.

In our study, we designed, built, and tested an autonomous CRISPR/Cas9-based gene drive system that genetically linked the Cas9 and sgRNA components to a fluorescent marker cargo such that the entire drive cassette was contained within the borders of the CRISPR/Cas9 target site. Similar to the original design of Gantz and Bier (2015) and the most recent design of Carballar-Lejarazú et al (2020), our focus was to position the gene drive system at a single genomic locus; however, we based the insertion locus on its capacity to be permissive for the expression of antiviral effectors. Of major concern to the development of an autonomous gene drive system with an associated genetically linked antipathogen 
effector is that the expression patterns for the HEG drive and the antipathogen effector are spatiotemporally distinct from each other. That is, the HEG drive is ideally active in the germline during early gametogenesis while the antipathogen effector is ideally expressed in tissues associated with the pathogen in the mosquitoes, such as in the female midgut. As a consequence, the genomic locus for an autonomous CRISPR/Cas9 gene drive must be permissive for both the expression of the gene drive as well as the antipathogen effector. Previous work using mariner/Mos1 transposable element (TE)mediated quasi-random integration of transgenes under control of the blood-meal inducible carboxypeptidase A promoter identified two genomic loci that exhibited strong transgene expression: 'TIMP-P4' and 'Carb109' (Franz et al., 2006; Dong et al., 2017). In addition, the Carb109 locus was found to be highly stable over 50 generations for a dengue virus type 2 (DENV2)-targeting inverted-repeat effector and represents an ideal genomic locus for the insertion and expression of antiviral effectors. Here, we investigated the potential to leverage genomic loci permissive for antiviral effector activity for the purposes of HEG-based gene drives using three promoters for Cas 9 expression in two different genome loci of Ae. aegypti.

\section{Materials and Methods}

\section{Generation of cDNA constructs}

The constructs used for establishing the gene drive lines were prepared in three sections: 1) an empty gene drive destination vector containing the homology arms corresponding to the destination locus (i.e.: TIMP-P4 or Carb109); 2) the NLS-Cas9 gene from the pHsp70-Cas9 plasmid (Addgene plasmid \#46294 and a gift from Melissa Harrison, Kate O'Connor-Giles \& Jill Wildonger; Wildonger et al., 2013) connected to the promoters and 3'UTRs from the B2tubulin (AAEL019894), nanos (AAEL012107), and innexin-4 (zpg, AAEL006726) genes, respectively, 3) the respective sgRNA for either the TIMP-P4 or the Carb109 locus under control of the U6:3 snRNA promoter (AAEL017774).

\section{Construction of empty destination vectors for the gene drive cassettes}

The empty destination vector for the TIMP-P4 locus (Chr2:32138225) was constructed as previously described for plasmid MT926371 from Williams et al. (2020), with the exception that the eCFP marker was replaced with that of mCherry as a two-fragment Gibson assembly. The ORF of mCherry was amplified using primers BR-100 and BR-101, while primers BR-98 and BR-99 were used to amplify the full backbone of the destination vector minus the eCFP marker (Table S1). The two PCR fragments were then assembled using the HiFi Gibson Assembly kit from NEB (Waltham, MA) following the manufacturer's 
instructions. The empty destination vector for the Carb109 locus (3: 409699138) was constructed by first cloning the 459 bp upstream homology arm using primers BR-368 and BR-112 (genomic positions AaegL5_3:409698681-409699140) using Kpnl and Xhol into pBluescript. The 541 bp right homology arm (genomic positions AaegL5_3: 3:409699139-409699680) was then amplified from the Ae. aegypti genome (Higgs' white eye (HWE) strain) using primers BR-113 and BR-364 and cloned into the left homology arm containing vector using Xhol and Sacll. Finally, the 3xP3-mCherry-SV40 cassette was amplified from the empty TIMP-P4 destination vector using primers BR-54 and BR-55 and cloned into the destination vector using $\mathrm{Xhol}$ and screened for orientation using primers BR-60 and BR-54.

\section{Construction of the B2tubulinCas9 cassette}

The basic components of the $\beta 2$ tubulinCas9 construct were assembled in pUC19 in three steps. First, the B2tubulin promoter/5'-UTR (AaegL5_2: 326339137-326338047) fragment was amplified from the Ae. aegypti genome (HWE strain) using primers BR-32 and BR-33 and cloned into pUC19 using Hindlll and Pstl, which introduced an Xhol site upstream of the promoter and an Ncol site at the end of the 5'-UTR of 82 tubulin. The 4272 bp ORF of Cas9 was then amplified from pHsp70-Cas9 plasmid (Addgene plasmid \#46294 and a gift from Melissa Harrison, Kate O'Connor-Giles \& Jill Wildonger; Wildonger et al., 2013) using primers BR-34 and BR-35 and inserted into the plasmid vector using Ncol and Sall. Finally the 3'UTR fragment of B2tubulin (AaegL5_2:326336649-326336454) was amplified using BR-28 and BR-29 and cloned into the assembly plasmid vector using Sall and $\mathrm{Xbal}$. The entire cassette was then removable from pUC19 using Xhol and Xbal for cloning into the TIMP-P4 or Carb109 destination vectors as either Xhol/Xbal insertions or Xhol/blunted insertions.

\section{Construction of the nanosCas9 cassette}

The 1159 bp nanos promoter fragment (AaegL5_1:228706-229865) was amplified from the HWE strain of Ae. aegypti using primers BR-40 and BR-41 and cloned into pBluescript using Xhol and screened for orientation using primers BR-99 and BR-41. The 4272 bp ORF of Cas9 was then amplified from pHsp70Cas9 plasmid (Addgene plasmid \#46294) using primers BR-34 and BR-35 and inserted into pBluescript downstream of the nanos promoter fragment using a Ncol and Sall digest for the PCR product and a Pcil / Sall digest for the plasmid vector. Finally, the 594 bp nanos 3'-UTR fragment (AaegL5_1:240330240924) was amplified from the Ae. aegypti genome (HWE strain) using primers BR-42 and BR-43 and cloned into the assembly plasmid vector downstream of the Cas9 ORF using Stul and Nhel. 


\section{Construction of Carb109-zpgCas9 $9^{G D}$}

The 1729 bp zpg promoter fragment (AaegL5_2:84862322-84863150) was amplified from genomic DNA of the HWE strain of Ae. aegypti using primers BR-655 and BR-656. The promoter region for the zpg gene in the HWE strain contained a 144 bp deletion (positions AaegL5_2:84863229-848632372), which was 825 bp upstream of the +1 ATG of the zpg ORF. The PCR product containing the promoter region was digested with $\mathrm{Xhol}$ and $\mathrm{Xbal}$ and cloned into pBluescript. In addition, primer BR-655 was internally tagged with a Bbsl site to allow for downstream scarless introduction of the N-terminus of the Cas9 ORF. The 1357 bp 3'UTR of the zpg gene (AaegL5_2:84865497-84866853) was amplified from Ae. aegypti (HWE strain) genomic DNA using primers BR-660 and BR-683 and cloned into the vector containing the zpg promoter using Pstl and Spel. In addition, primer BR-660 was internally tagged with an inverted BbsI site to allow for downstream scarless introduction of the C-terminus of the Cas9 ORF. Subsequently, the 4272 bp ORF of Cas9 was then amplified from pHsp70-Cas9 plasmid (Addgene plasmid \#46294) using primers BR-666 and BR-667 and cloned into the vector between the zpg promoter and its 3'UTR using Bbsl for scarless, directional cloning.

Assembly of the Carb109-3xP3-eCFP eye marker vector

The Carb109-3xP3-eCFP vector was constructed by replacing the mCherry ORF from the Carb109 destination vector with eCFP. The eCFP ORF was amplified from the MT926371 from Williams et al. (2020) using primers BR-100 and BR-101. The mCherry-deleted backbone from the empty Carb109 destination vector was amplified using BR-98 and BR-99. The two PCR fragments were then assembled using the HiFi Gibson Assembly kit from NEB (Waltham, MA) following the manufacturer's instructions.

\section{Assembly of the Pol III promoter / chiRNA empty vector}

The empty Pol III promoter / chiRNA cassette was synthesized as a gBlock (IDT, Corralville, IA) starting with the promoter region for AAEL017774 (AaegL5_3:382122755-382123154, minus strand) with a G to A substitution at the -6 position relative to the TATA box in order to domesticate a Bbsl restriction site and allow for Golden-Gate cloning (Engler et al., 2008). The sgRNA scaffold from Dang et al. (2015) was placed downstream of the promoter and separated by two inverted $\mathrm{Bbsl}$ sites oriented such that the generated 5' overhang regions were present within the promoter and chiRNA respectively. Primers tagged with Sacll restriction sites were used to PCR amplify the full gBlock CDNA, which was then cloned into pBluescript using Sacll. Subsequently, the sgRNA programming for all gRNA was prepared by digesting the empty Pol III promoter / chiRNA vector with Bbsl followed by ligation of the respective 
adapter primers (BR- 360 and BR-361 for the TIMP-P4 locus, BR-362 and BR-363 for the Carb109 locus, and BR-358 and BR-359 for the sgRNA cassette) to target the EGFP disrupted ORF in the pCas9activity plasmid. Adapters were unphosphorylated and tagged with 5'-AAAT for the protospacer sequence (PAM-sense strand) and 5'-AAAC for the corresponding reverse complement (PAM antisense strand), following the approach outlined in Gokcezade et al. (2014). The respective programmed U6-sgRNA cassettes were then amplified using BR-350 and BR-351 and cloned into the destination vectors using Sacll followed by screening for orientation using the appropriate forward adapter (BR-360 for TIMP-P4 and BR-362 for Carb109) and M13R primers.

\section{Assembly of the PUbCas9SV40term construct}

The polyubiquitin promoter (PUb, AAEL003888) from Anderson et al. (2010) was amplified from the Ae. aegypti (HWE) genome (AaegL5_3: 84657201-84658584) and cloned into the pBluescript phagemid vector using $\mathrm{Xhol}$ and Notl. The Cas9 ORF along with the transcription terminator from the Simian virus 40 (SV40) large T antigen was then amplified from the pBS-Hsp70-Cas9 plasmid (Addgene plasmid \#46294) using primers tagged with Notl and Sacll and inserted downstream of the Pub promoter fragment.

\section{Assembly of the PUbEGFPSV40term construct}

The positive control plasmid for EGFP expression for the C6/36 transfection assay to test for U6 promoter activity was constructed by replacing the Cas9 ORF from the PubCas9SV40term plasmid with the ORF from EGFP. The EGFP ORF cDNA was ordered as a gBlock from IDT (Corralville, IA), amplified using BR-100 and BR-101, and digested with Ncol and Notl. The digested PCR product was then cloned into the PubCas9SV40term plasmid using Ncol and Notl, which also excised the Cas9 ORF.

\section{Generating the pAeT7ku70 plasmid for dsRNA production}

A total of 10 fourth instar Ae. aegypti (HWE strain) larvae were collected, flash frozen on dry ice and then extracted for total RNA using TRIzol reagent (Carlsbad, CA). First strand cDNA synthesis was performed using the Protoscript cDNA kit from NEB (Waltham, MA) following the manufacturer's instructions. The ku70 dsRNA template published in Basu et al. (2015) was then amplified from the cDNA using primers BR-44 and BR-45, which tagged both ends of the PCR product with the T7 promoter. Subsequently, the T7-tagged PCR product was amplified using Hindlll-tagged T7 (BR-80) and cloned into pUC19 using HindIII. 


\section{Final assemblies of gene drive constructs}

The final assemblies of the gene drive constructs were prepared using a three-step cloning strategy. Cas9 cassettes for the TIMP-P4 or the Carb109 locus were cloned into their respective destination vectors using $\mathrm{Xhol} / \mathrm{Sacll}$, followed by the addition of the mCherry fluorescent marker under control of the 3xP3 synthetic promoter (Horn et al., 2000) using Xhol, and finally the addition of the appropriate U6 promoter plus sgRNA using Sacll. The schematic for our gene drive constructs is presented in Figure S1, and the primers used in our study are listed in Table S1. The final sequences for the constructs are available at NCBI as outlined in Table S2.

\section{Generation of transgenic lines}

\section{Mosquito rearing}

The HWE strain of Ae. aegypti was used for the generation of all transgenic lines in our study. The larval stages were reared in deionized water at $28^{\circ} \mathrm{C}$ with a $12: 12$ (L:D) photoperiod and fed a diet of Tetramin Tropical flakes (Tetra Spectrum Brands Pet, LLC, Blacksburg, VA). Pupae were transferred to plastic containers and allowed to emerge in 18 cubic-inch cages and provided with deionized water and raisins as food sources.

\section{CRISPR/Cas9 target site identification}

For our initial testing, we first assessed the activities of six sgRNAs for activity proximal to the Carb109 genome locus, while the active sgRNA for the TIMP-P4 genome locus was already reported (Williams et al., 2020). To accomplish this, we prepared all injection mix material so that the final concentrations of Cas9-NLS protein was $300 \mathrm{ng} / \mu \mathrm{L}$ and the concentration of sgRNA was $80 \mathrm{ng} / \mu \mathrm{L}$. Then, three sets of approximately 100 pre-blastoderm embryos were injected with injection mix and incubated overnight $(18-24 \mathrm{~h})$ at $27^{\circ} \mathrm{C}$ before being homogenized and extracted for gDNA into each of three pools. The genomic sequence of the Carb109 locus was then PCR amplified using primers BR-60 and BR-65, and the resulting amplicons gel-purified using the Zymo Clean and Concentrator kit (Zymo Research, San Diego, CA). PCR products were subsequently sequenced at the University of Missouri DNA Core and analyzed visually for trace decay at the predicted CRISPR/Cas9 cleavage site. All products were sequenced in both directions to confirm the correct site of sequence decay. 


\section{Harvest and microinjection of embryos}

Cages of 7-10 d-old mosquitoes were provided with a blood meal (defibrinated sheep blood, Colorado Serum, Denver, $\mathrm{CO}$ ) and maintained at $28^{\circ} \mathrm{C}$ under a $12: 12$ (L:D) photoperiod for four days prior to injection. Groups of 15-20 hyper-gravid females were then mouth aspirated into an aluminum foilwrapped $50 \mathrm{~mL}$ conical tube containing two $2.5 \times 0.5 \mathrm{~cm}$ strips of moistened, overlapping Whatman \#1 filter paper and allowed to oviposit for $20 \mathrm{~min}$. Early embryos were then aligned over a period of 20 to 30 min using a fine spotter paint brush such that all posterior ends of the embryos faced the same direction. Aligned embryos were then transferred to double-faced tape (Scotch brand, 3M Columbia, MO) affixed to a plastic coverslip (Fisher Scientific, Waltham, MA) and covered with a layer of Halocarbon 27 oil (Sigma-Aldrich, St. Louis, MO) prior to the visible onset of melanization. Embryos were then injected with injection mix using a pulled and beveled micropipette capillary tube (Sutter Instruments, Novato, CA) connected to a Femtojet 5247 air compressor (Eppendorf, Germany) at 650 psi positive pressure and 100 psi backpressure. Immediately following injection, the Halocarbon 27 oil was gently washed from the embryos with deionized water, and the embryos were allowed to age in a humid chamber for a minimum of $2 \mathrm{~h}$ prior to transfer to a $500 \mathrm{~mL}$ plastic beaker lined with 5-10 layers of moistened Kimwipes. Injected embryos (herein referred to as G0) were then maintained in the humid plastic beaker for $6 \mathrm{~d}$, transferred to a small plastic cup filled with deionized water and allowed to hatch out. GO survivors were reared as described above and backcrossed to wildtype HWE. For the backcrossing, male G0 individuals were individually provided with 5-10 virgin females and allowed to mate for 3-5 days, then pooled into larger cartons using 20 of the smaller cartons for each large carton. Female G0 were collected into large cartons (up to 100 per carton) and provided with half the number of virgin HWE males and allowed to mate for 3-5 days. All cages were then provided with a minimum of three blood meals (defibrinated sheep blood, Colorado Serum) and allowed to lay eggs onto filter papers. G1 eggs were allowed to mature for five days and were then hatched, reared to fourth instar, and screened for the presence of the fluorescence marker. Positive G1 individuals were subsequently outcrossed to HWE again to establish the transgenic lines. Integration of the transgene was validated by PCR, and Sanger sequencing using primers BR-13 and BR-115 for transgenes at the Carb109 locus and primers BR-51 and BR-73 at the TIMP-P4 locus.

\section{Genetic crosses to test for gene drive activity}

An initial pool of G0 hemizygous individuals was established for each of the gene drive lines by outcrossing 50 transgenic males to 200 HWE virgins en masse and allowing them to mate and take a 
blood meal. The G0 eggs were subsequently collected and used as the starting material for each of the drive activity crosses in our study. From the G0 pools, a total of 12 hemizygous males were individually crossed to two virgin HWE females, and 20-25 hemizygous females were crossed to an equal number of male HWE en masse and allowed to mate for 3-5 days. The individual male containers were each provided with their own blood meals, while the female container was provided with a common blood meal, followed by the transfer of blood-fed females to individual cartons for egg laying. Following oviposition, egg papers were removed and allowed to develop for a minimum of five days prior to hatching individually into 4 oz plastic cups. Larvae were reared to third/fourth instar and scored for the presence of the transgenic marker to determine the level of gene drive inheritance. Following the scoring of the outcross-1 (OX-1) mosquitoes, two pools of crosses representing either a 'low' level (i.e.: Mendelian-like), or 'high' level (i.e.: Super-Mendelian-like) were selected from each cross such that an additional 12 female and 12 male G1 hemizygotes could be outcrossed again to HWE for the OX-2 assessment. The selection of the 'low' and 'high' pool, therefore, did not necessarily represent the lowest and highest levels of gene drive inheritance, but rather served as representative pools from which to establish the following outcross. From within OX-1, non-transgenic individuals were saved to assess for the presence of GDBI. For the AeaNosC109 ${ }^{\mathrm{GD}}$ and $\mathrm{ZpgNosC} 109^{\mathrm{GD}}$ lines, all non-transgenic mosquitoes were selected for assessment, while for the AeaNosT4 ${ }^{\mathrm{GD}}$ and Aeaß2tC109 ${ }^{\mathrm{GD}}$ lines, a total of 20 and 10 individuals were selected, respectively. All crosses were duplicated and the data pooled for analysis.

\section{Statistical analyses}

Comparisons of the OX-1 gene drive levels for the lines were assessed in R using a Kruskal-Wallace nonparametric ANOVA followed by a Dunn-Bonferroni post hoc means separation procedure to test for significance across all groups and all lines. Comparisons of the OX-2 gene drive levels, and for the levels of gene drive blocking indels, were assessed in $\mathrm{R}$ using a Kruskal-Wallace nonparametric ANOVA followed by a Dunn-Bonferroni post hoc means separation procedure to test for significance for all groups within each line individually. Comparisons of the maternal and paternal contributions were assessed using pairwise T-testing in R following a Shapiro-Wilk test for normality. Deviation from the expected Mendelian inheritance level for the eCFP marker in the paternal contribution testing was conducted manually using Chi-Square analysis. 


\section{Testing of paternal contribution of Cas9-RNP}

Initial crosses to establish trans-hemizygous mosquitoes were conducted using AeaNosC $109^{\mathrm{GD}}$ females outcrossed to males harboring a 3XP3-eCFP-SV40 fluorescent eye marker at the Carb109 locus. The positionings of the fluorescent markers in both transgenes were in opposite orientation to one another to mitigate the potential for crossing over. Following this cross, fourth instar progeny were screened and selected for the presence of both an mCherry (gene drive) and an eCFP (null drive) marker and reared to adults. Subsequently, virgin hemizygous females or virgin hemizygous males were outcrossed to wildtype HWE following the methodology described in the previous section describing genetic crosses to test for gene drive activity. The progeny from this cross were then scored for the numbers of offspring containing either mCherry or an eCFP eye marker to test for the possibility of a fitness cost at the germline level relative to the eCFP (null drive) marker. The females from each of these crosses were saved (within cross) and subsequently mated again to an equal number of HWE males, provided with a single blood meal after which females were separated to allow them to lay eggs individually. Eggs were allowed to mature for at least $5 \mathrm{~d}$ and were then hatched and scored for the presence of the eCFP marker to determine the level of transgene inheritance. In addition, five non-transgenic larvae were selected from each of the pools for the assessment of GDBI.

\section{Assessment of GDBI}

GDBI were assessed as inheritable refractory indels by assessing the nucleotide sequence of the alternative inheritable allele in outcrossed hemizygous individuals. For the OX-1 generation of the gene drive assessment assays, non-transgenic mosquitoes were individually analyzed by PCR using BR-20 and BR-23 for the TIMP-P4 locus, and BR-60 and BR-65 for the Carb109 locus. PCR products were amplified, purified using the Zymo Clean and Concentrator kit (Zymo Research, San Diego, CA) and Sanger sequenced. Chromatogram traces were then assessed manually for trace decay at the predicted CRISPR/Cas9 cleavage site. For the assessment of GDBI in the OX-2 generation, all non-transgenic mosquitoes within a cross were pooled and their DNA extracted to obtain an 'average' number of GDBI for the non-drive-inheriting mosquitoes. PCR products were then amplified using BR-724 and BR-725 for the Carb109 locus and BR-726 and BR-727 for the TIMP-P4 locus to obtain the 'round 1' PCR products for Illumina whole-amplicon sequencing. The first round PCR was held to 30 cycles. PCR products were subsequently purified using the Zymo Gel Extraction kit (Zymo Research, San Diego, CA) and adjusted to $1 \mathrm{ng} / \mathrm{uL}$ using a Qubit fluorimeter (ThermoFisher Scientific, Waltham MA), and material was subsequently sequenced using paired end reads of 250 bp length (PE250) on an Illumina MiSeq 
instrument. The resulting reads were trimmed using Cutadapt v1.01 (Martin, 2011) and assessed for indels using Crispresso v2 (Clement et al., 2019).

\section{Results}

Identification of active target sites and target site polymorphism

In our previous work (Williams et al., 2020), we identified a highly active sgRNA for the TIMP-P4 locus. In our current work, we identified a novel active CRISPR/Cas9 target site for the Carb109 locus located 1214 bp downstream of the original mariner/Mos1 insertion site at the Carb109 locus (Franz et al., 2014). Since the original Carb109 mariner/Mos1 insertion was determined to be within the 3'-UTR of the single-copy polyadenylate-binding protein gene (AAEL010318), we manually selected CRISPR/Cas9 targets as close to, but not within, the AAEL010318 gene and assessed for off-targets using CHOPCHOP V2 (Labun et al., 2016). In total, we assessed six sgRNA for activity (Table 1) and identified only a single sgRNA (\#15) that showed DNA cleavage activity. Therefore, for all experiments at the Carb109 locus, sgRNA \#15 was selected.

Table 1. Active CRISPR/Cas9 target sites proximal to the original TIMP-P4 and Carb109 mariner/Mos1 insertion loci.

\begin{tabular}{|c|c|c|c|}
\hline Locus & sgRNA number & $\begin{array}{l}\text { Genomic locus (distance from } \\
\text { original Mos1 insertion site) }\end{array}$ & $\begin{array}{l}\text { Activity (\# pools / pools } \\
\text { sampled) }\end{array}$ \\
\hline TIMP-P4 & sgRNA \#5* & $2: 321382225 \quad(623 \mathrm{bp})$ & Active $(3 / 3)$ \\
\hline \multirow[t]{6}{*}{ Carb109 } & 2 & 3: 409717866 (19942 bp) & Not active $(0 / 3)$ \\
\hline & 4 & 3: 409723215 (25291 bp) & Not active $(0 / 3)$ \\
\hline & 6 & 3: 409699241 (1317 bp) & Not active $(0 / 3)$ \\
\hline & 15 & 3: 409699138 (1214 bp) & Active $\quad(3 / 3)$ \\
\hline & 21 & 3: 409699885 (1961 bp) & Not active $(0 / 3)$ \\
\hline & 29 & 3: 409699526 (1602 bp) & Not active $(0 / 3)$ \\
\hline
\end{tabular}

*Previously reported in Williams et al., 2020

In addition to the assessment of sgRNA activity, we analyzed the genomic target sites for polymorphisms using the data from Schmidt et al. (2020), which examined CRISPR/Cas9 target sites across 132 genomes of Ae. aegypti. Two polymorphisms were found in the TIMP-P4 locus: one in the PAM that changed the second base $\mathrm{G}$ to a $\mathrm{T}$ and had an allele frequency of 0.026 among the 132 genomes tested, while the second polymorphism was at position 13 distal to the PAM that changed the base from a $\mathrm{G}$ to a $\mathrm{C}$ at an allelic frequency of 0.009 (Table 2). Interestingly, the Carb109 locus contained only a single polymorphism at position 19 distal to the PAM that still allows for the use of an 18 bp protospacer, which is considered functional for Cas9 activity (Cencic et al., 2014). 
Table 2. Polymorphisms in the active target sites for the Carb109 and TIMP-P4 loci among 132 genomes of Aedes aegypti. The polymorphisms are indicated in boldface, while the PAM sequences are underlined.

\begin{tabular}{|c|c|c|c|c|}
\hline SgRNA & $\begin{array}{c}\text { Polymorphisms } \\
\left(5^{\prime} \text {-protospacer + PAM -3') }\right.\end{array}$ & $\begin{array}{c}\text { Genomic } \\
\text { locus* }\end{array}$ & $\begin{array}{l}\text { Genome } \\
\text { coverage }\end{array}$ & $\begin{array}{c}\text { Polymorphism } \\
\text { frequency }\end{array}$ \\
\hline \multirow[t]{2}{*}{ TIMP-P4 } & GACCAACGGCAGTCATTGTG ITG & $2: 321382218$ & 230 & 0.026087 \\
\hline & GACCAACCGCAGTCATTGTG $\underline{\text { TGG }}$ & $2: 321382231$ & 222 & 0.00900901 \\
\hline Carb109 & GWTATGCCGAAGAAAAGCCA GGG & 3:409699154 & 264 & 0.0113636 \\
\hline
\end{tabular}

Embryo injection survival and knock-in rates

A total of six transgenic lines were established with an embryo injection effort ranging from $568-1442$ embryos (Table 3). In general, the survival rate of the injected G0 mosquitoes ranged from a low of 3.9\% (AeaeCFPCarb109) to a high of 23\% (AeaNosCarb109GD). In addition, we identified that the nanosCas9 constructs required no helper Cas9 or helper sgRNA in order to integrate into the genome. Regardless, after the initial successful injection of the nanosCas9 construct into the TIMP-P4 locus, helper Cas9 protein was added to all injection mixes, with the omission of the helper sgRNA and continued inclusion of the ku70 dsRNA trigger.

Table 3. Aedes aegypti lines used in our study and respective injection efforts for their establishment.

\begin{tabular}{|c|c|c|c|c|}
\hline Line name & Marker & Purpose & $\begin{array}{l}\text { Embryos } \\
\text { injected }\end{array}$ & Survivors (F:M ; \%) \\
\hline Aeaß2tTIMP-P4 ${ }^{G D}$ & $3 \times \mathrm{P} 3$ mCherry & Gene drive at TIMP-P4 locus & 1115 & $72(31: 41 ; 6.5 \%)$ \\
\hline AeaNosTIMP-P4 $4^{G D}$ & $3 \times \mathrm{PP}$ mCherry & Gene drive at TIMP-P4 locus & 959 & $154(66: 88 ; 16 \%)$ \\
\hline Aeaß2tCarb109GD & 3xP3 mCherry & Gene drive at Carb109 locus & 882 & $122(64: 58 ; 13 \%)$ \\
\hline AeaNosCarb109GD & $3 \times \mathrm{x} 3$ mCherry & Gene drive at Carb109 locus & 912 & 206 (90:116 ; 23\%) \\
\hline AeaZpgCarb109GD & 3xP3 mCherry & Gene drive at Carb109 locus & 568 & 45 (17:28; 7.9\%) \\
\hline AeaeCFPCarb109 & $3 \times P 3$ eCFP & $\begin{array}{l}\text { Genetic balancer for Carb109 } \\
\text { locus }\end{array}$ & 1142 & $44(24: 20 ; 3.9 \%)$ \\
\hline
\end{tabular}

Gene drive levels

Overall, the strongest gene drive activity was observed when Cas9 was expressed from the nanos promoter at the Carb109 locus in the AeaNosCarb109 ${ }^{\mathrm{GD}}$ line, where for the first outcross, drive levels as 
high as $94 \%(n=55 / 59)$ and $96 \%(n=74 / 77)$ were identified for the female and male groups, respectively (Figure 1; Table S2). The median values for marker inheritance demonstrated no gene drive activity for the Aeaß2tCarb109 ${ }^{\mathrm{GD}}$ line, with transgene inheritance levels similar to expected Mendelian inheritance levels, ranging from a low of $43 \%$ in male parentals $(n=24 / 56$ ) to a high of $55 \%$ in both male $(n=16 / 29)$ and female $(n=11 / 20)$ parental groups.

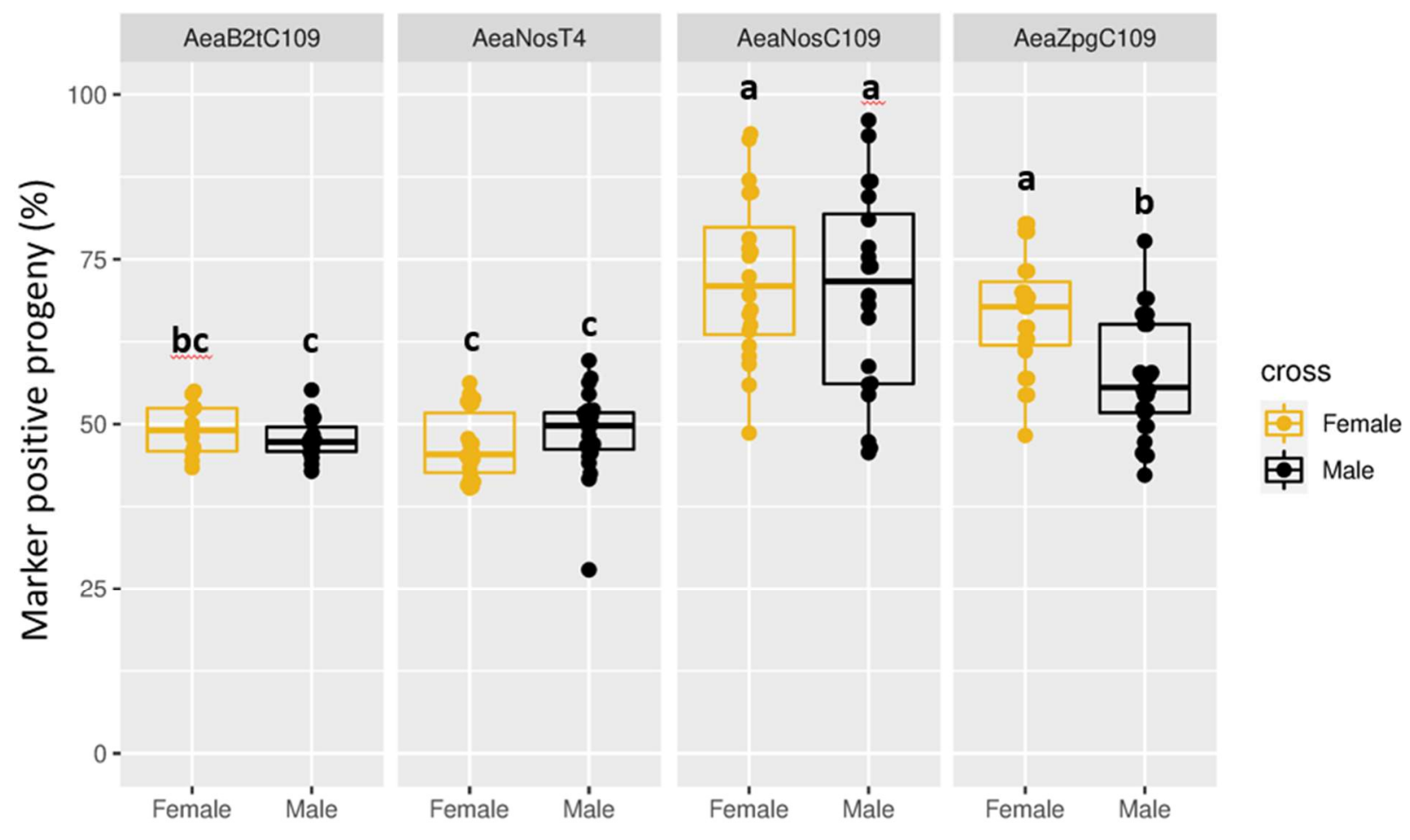

Parental transgenic sex for the outcross

Figure 1. Frequency of marker inheritance in first outcrossed (OX-1) individuals from hemizygous outcrossed females or males for gene drive (GD) lines with GD insertions at two different genomic loci (Carb109 or TIMP-P4) and Cas9 expression being controlled by three different promoters ( $\beta 2$ tubulin, nanos, or zpg). The bar internal to the box represents the median value, while the lower and upper borders of the boxes represent Q1 and Q3, respectively. Groups superseded with the same letter are not statistically different $(p>0.05)$. Each data point represents the percentage of transgene inheritance resulting from the offspring of the parental crosses where each transgenic female parental was allowed to mate to two wildtype males, and each transgenic male parental was allowed to mate to two wildtype females. A minimum of 20 larvae was set for each group in order to be scored. For female parental crosses, the population sizes of each data point ranged from 20 to 142 larvae (mean \pm SEM $=59 \pm 3$ larvae). For male parental crosses, the population sizes of each data point ranged from 21 to 415 larvae (mean \pm SEM $=91 \pm 5$ larvae).

Given that the Carb109 locus was permissive for gene drive activity, we tested another promoter, zero population growth (zpg), which has been shown have strong gene drive activity in An. gambiae but has 
not been tested in Ae. aegypti (Hammond et al., 2016; Kyrou et al., 2018). Overall, gene drive activity was observed for the AeaZpgCarb109 ${ }^{\mathrm{GD}}$ line at the Carb109 locus, with significant gene drive levels similar to the AeaNosCarb109 ${ }^{G D}$ line for female transgenic parentals. Male transgenic parentals of the AeaZpgCarb109 ${ }^{G D}$ line, however, had a significantly lower $(p=0.0258)$ level of gene drive when compared with either parental cross for the AeaNosCarb109 ${ }^{\mathrm{GD}}$ line, or with female transgenic parentals of the AeaZpgCarb109 ${ }^{\mathrm{GD}}$ line. These results showed that while the $z p g$ promoter is active in Ae. aegypti, there may be a sex-specific effect on Cas9 expression, which was unanticipated for this promoter since the zpg gene (innexin-4) is involved in early germ cell development in both male and female Drosophila (Tazuke et al., 2012). Given that the Aeaß2tCarb109 ${ }^{G D}$ line did not demonstrate gene drive activity, we did not continue with this line for further assessment at the OX-2 level. Regardless, we continued with the assessment of the AeaNosT4 ${ }^{G D}$ line since the gene drive cargo was identical to that of the AeaNosCarb109 ${ }^{G D}$ line, although no gene drive activity was observed. This allowed us to test for the effect of genomic insertion locus on the activity of a (single component) autonomous CRISPR/Cas9 gene drive system in Ae. aegypti.

Regarding the OX-2 crosses, we were interested in answering two questions: 1) Would the effects of 'low' and 'high' levels of gene drive inheritance from the OX-1 crosses be consistent? and 2) Do GDBI accrue differently for female and male transgenic parentals? We assessed for this by first identifying individual crosses from each of the OX-1 groups that represented 'low' and 'high' levels of gene drive (Table 4) and subsequently outcrossed transgenic female and males reciprocally to HWE mosquitoes as was performed in OX-1. This resulted in a total of eight crossing groups within each line, where we coded the crosses with three-letter codes that identified the parental ( $F$ 'female' or $M$ 'male'), the grandparental ( $F$ 'female' or $M$ 'male'), and the level of gene drive observed for the OX-1 pool ( $L$ 'low' or $H$ 'high'). When the progeny ( $\mathrm{n}$ ranging from 795 to 3162 ) for all of the OX-2 crosses were scored, we again observed Mendelian-like inheritance of the gene drive cassette for the AeaNosT4 ${ }^{\mathrm{GD}}$ line. However, one cross in the $\mathrm{FFH}$ group resulted in $90 \%$ inheritance $(n=124)$, suggesting that while gene drive at the TIMP-P4 locus was generally non-permissive for gene drive using the nanos promoter for Cas9 expression, occasional cases of gene drive might still occur (Figure 2; Table S3). The results for the AeaZpgC109 ${ }^{G D}$ line displayed similar levels of gene drive for all 157 crosses, with five pools of crosses demonstrating $>80 \%$ levels of transgene inheritance and the weighted average levels of inheritance greater than $50 \%$ for all crosses. The median values, however, had an inheritance of $<50 \%$ for the $\mathrm{MMH}$ group, which incidentally also included the cross with the highest level of transgene inheritance among 
all the crosses. These results suggested that the effect of 'low' and 'high' levels of gene drive were not inheritable by the OX-2 generation. Finally, for the AeaNosC109 ${ }^{G D}$ line, four of the crosses, MMH, MFH, $F F L$, and MFL, contained individual crosses ( $n=20,44,114$, and 137, respectively) that resulted in complete (100\%) transgene inheritance. In addition, the levels of gene drive were the same $(p>0.05)$ for male parentals with any of the grandparental combinations, whether originating from 'low' or 'high' levels of gene drive. In the female parental crosses, however, a significant decrease in the level of gene drive was observed for the FML cross, which was unexpected since none of the other 'low' level crosses displayed a 'low' level of gene drive in the OX-2. We therefore conducted assessment of all the nontransgenic mosquitoes (219 pools, average pool size $=26.2 \pm 1.3$ larvae, total $n=5732$ ) in the OX-2 pools using whole amplicon sequencing to determine the levels of GDBI. The presence of those indels could explain the reduced inheritance level of the gene drive in the AeaNosC109 ${ }^{\mathrm{GD}}$ crosses, particularly in the FML crosses, which demonstrated the greatest range of transgene inheritance $(22.9-93.8 \%)$ and had the lowest weighted mean of all of the AeaNosC109GD crosses $(57.4 \pm 24.0 \%)$.

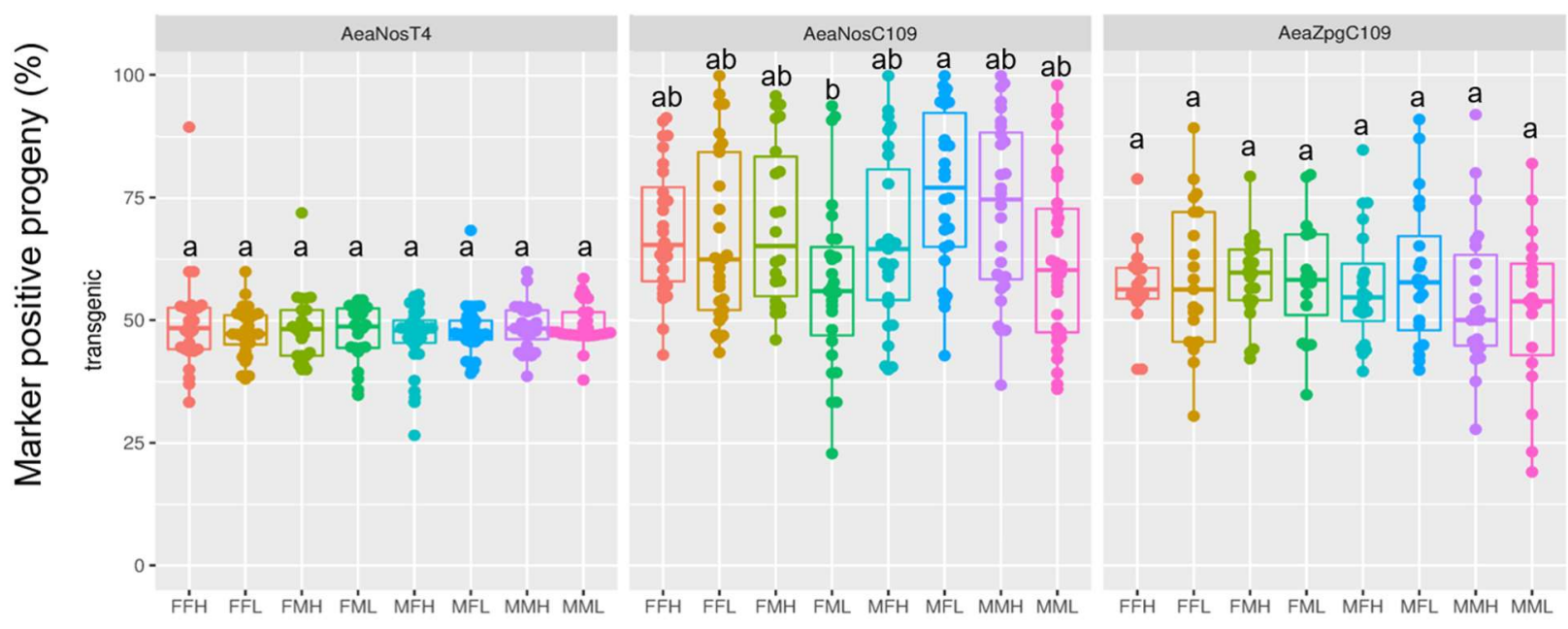

Parental/Grandparental transgenic sex for the outcross

Figure 2. Frequency of marker inheritance in progeny from second-outcrossed (OX-2) individuals (594 groups, average $n=72 \pm 2$, total $n=43,015$ ) originating from either 'high' or 'low' parental drive pools with GD insertions at two different genomic loci ("C109" for Carb109 or "T4" for TIMP-P4) and Cas9 expression being controlled by two different promoters ("Nos" for nanos or "Zpg" for zero population growth). The bar internal to the box represents the median value, while the lower and upper borders of the boxes represent Q1 and Q3, respectively. Groups within gene drive line that are superseded with the same letter are not statistically different $(p>0.05)$. The first letter of each cross designation indicates the OX-2 parental transgenic sex and the second letter indicates the OX-1 grandparental transgenic sex, where $F=$ female and $M=$ male. $L=$ low drive level in the grand parental generation, $H=$ high drive in the parental generation. Each data point represents the percentage of transgene inheritance resulting from the offspring of the parental crosses where each transgenic female parental was allowed to mate with two wildtype males, and each transgenic male parental was allowed to mate with two wildtype females. A minimum of 20 larvae was set for each group in order to be scored. 
Gene drive blocking indels (GDBI)

Overall, the occurrence of GDBI in the OX-1 crosses was most pronounced in the AeaNosC109GD line

(Table 4).

Table 4. Testing of non-transgenic offspring from OX-1 individuals to assess for the presence of gene drive blocking indels (GDBI) among the gene drive lines.

\begin{tabular}{|c|c|c|c|}
\hline Line & Drive Parental & $\begin{array}{c}\text { Drive rate } \\
\text { (\% marker inheritance; } n \\
\text { total) }\end{array}$ & $\begin{array}{c}\text { Number of GD-negative } \\
\text { larvae with indels / total } \\
\text { number of GD negative larvae } \\
\text { assessed (\%) }\end{array}$ \\
\hline \multirow[t]{4}{*}{ AeaNosT4 ${ }^{G D}$} & Male & Low (45.9\%; 290) & $0 / 40(0 \%)$ \\
\hline & & High (58.1\%; 136) & $0 / 40(0)$ \\
\hline & Female & Low $(42.9 \% ; 163)$ & $0 / 40(0)$ \\
\hline & & High (51.3\%; 117) & $0 / 40(0)$ \\
\hline \multirow[t]{4}{*}{ AeaNosC109 ${ }^{G D}$} & Male & Low $(55.3 \% ; 228)$ & $9 / 54(16.7)$ \\
\hline & & High $(86.4 \% ; 214)$ & $7 / 27(25.9)$ \\
\hline & Female & Low $(61.5 \% ; 148)$ & $7 / 46(15.2)$ \\
\hline & & High (89.7\%; 136) & $7 / 12(58.3)$ \\
\hline \multirow[t]{4}{*}{ AeaZpgC109GD } & Male & Low $(56.5 \% ; 232)$ & $0 / 41(0)$ \\
\hline & & High (59.2\%; 228) & $0 / 51(0)$ \\
\hline & Female & Low $(57.1 \% ; 98)$ & $2 / 27(7.4)$ \\
\hline & & High $(73.7 \% ; 118)$ & $1 / 23(4.3)$ \\
\hline \multirow[t]{2}{*}{ Aeaß2tC109 } & Male & no drive $(50.7 \% ; 138)$ & $0 / 10(0)$ \\
\hline & Female & no drive $(52.5 \% ; 40)$ & $0 / 10(0)$ \\
\hline
\end{tabular}

Meanwhile, the zpg promoter similarly displayed some GDBI, albeit at a much lower level (7.4\% for 'low' drive individuals, and $4.3 \%$ for 'high' drive individuals), and only in pools of offspring originating from females. For both the Aeaß2tC109 ${ }^{G D}$ and AeaNosT4 ${ }^{G D}$ lines, no GDBI were identified; however, these lines demonstrated normal Mendelian-like inheritance of the gene drive; thus, it was not expected that GDBI would arise in these lines. Given that the GDBI identified in the OX-1 crosses were inherited from the original male transgenic to HWE females in the OX-0 cross, we further assessed the levels of GDBI for the OX-2 crosses to identify if there were differences between female and male groups with respect to the number of GDBI that arose in the population (Figure 3). Overall, we identified negligible proportions (<5\%) of amplicons containing GDBI in the AeaNosT4 ${ }^{G D}$ line, comparable to the single HWE control sample (Figure 3). For the AeaNosC109 ${ }^{\mathrm{GD}}$ line, all crossing groups displayed variable proportions of amplicons containing GDBI. In addition, we observed nine crosses for which the proportion of amplicons containing GDBI were $>50 \%$, which is the theoretical maximum for amplicons containing 
indels when considering outcrossed gene drive negative larvae. Of these nine crosses, two data points were close to $50 \%$ (FFL: $54 \%$, MFL: $53 \%$ ), while the remaining seven data points ranged from $64 \%$ ( $\mathrm{MMH}$ ) to $100 \%$ (MML). These higher levels of identified amplicons with GDBI could be an artifact of chimeric PCR. Thus, our analysis of pooled larvae using the high throughput sequencing was likely overestimating the actual number of GDBI in the sample. One group within the AeaNosC109GD line, MFH, contained very few amplicons showing the presence of indels (median =14.5\%); however, the median proportion of amplicons containing indels was not significantly lower in this group than in the other groups within the AeaNosC109 ${ }^{G D}$ line $(p=0.0831)$. Finally, in the AeaZpgC109 ${ }^{G D}$ line, a significantly greater median number of GDBI was observed for the FFL group (median $=2.6 \%$ ) when compared to the $\mathrm{MMH}$ (median $=0.02 \%$ ) group. In general, all male parental crosses appeared to have fewer GDBI (median range $=0.02-1.57 \%)$ than the female parental crosses (median range $=1.97-11.85 \%)$, regardless of the sex of the grandparental transgenic individual. This result was unexpected because in Drosophila, the zpg gene is active in both the female and male pregonadal stem cells (Tazuke et al., 2002). Interestingly, there was no apparent grandparental effect for the zpg promoter-driven Cas9 gene drive. In addition to the abundance of GDBI assessed for the OX-2 cross, we also assessed the size distributions of the resultant insertions/deletions (Figure S1). Overall, deletions were the most abundant type of indel, representing $86 \%, 94 \%$, and $97 \%$ of all indels for the AeaNosT4 ${ }^{\mathrm{GD}}$, AeaNosC109 ${ }^{\mathrm{GD}}$, and AeaZpgC109 ${ }^{G D}$ lines, respectively. Nearly all identified deletions were present within $50 \mathrm{bp}$ of the CRISPR/Cas9 target site, with the largest deletion being 82 bp in the AeaZpgC109 ${ }^{G D}$ line. The largest identified insertion was $60 \mathrm{bp}$ in the AeaNosC109 ${ }^{\mathrm{GD}}$ line, although nearly $100 \%$ of the insertions in all lines tested were within $50 \mathrm{bp}$ of the CRISPR/Cas9 target site. Taken together, these results suggested that for the purposes of an autonomous gene drive system in Ae. aegypti, the homologous region required for strand invasion of the cleaved DNA proximal to the predicted CRISPR/Cas9 target is likely a minimum of around $50 \mathrm{bp}$ in size. 

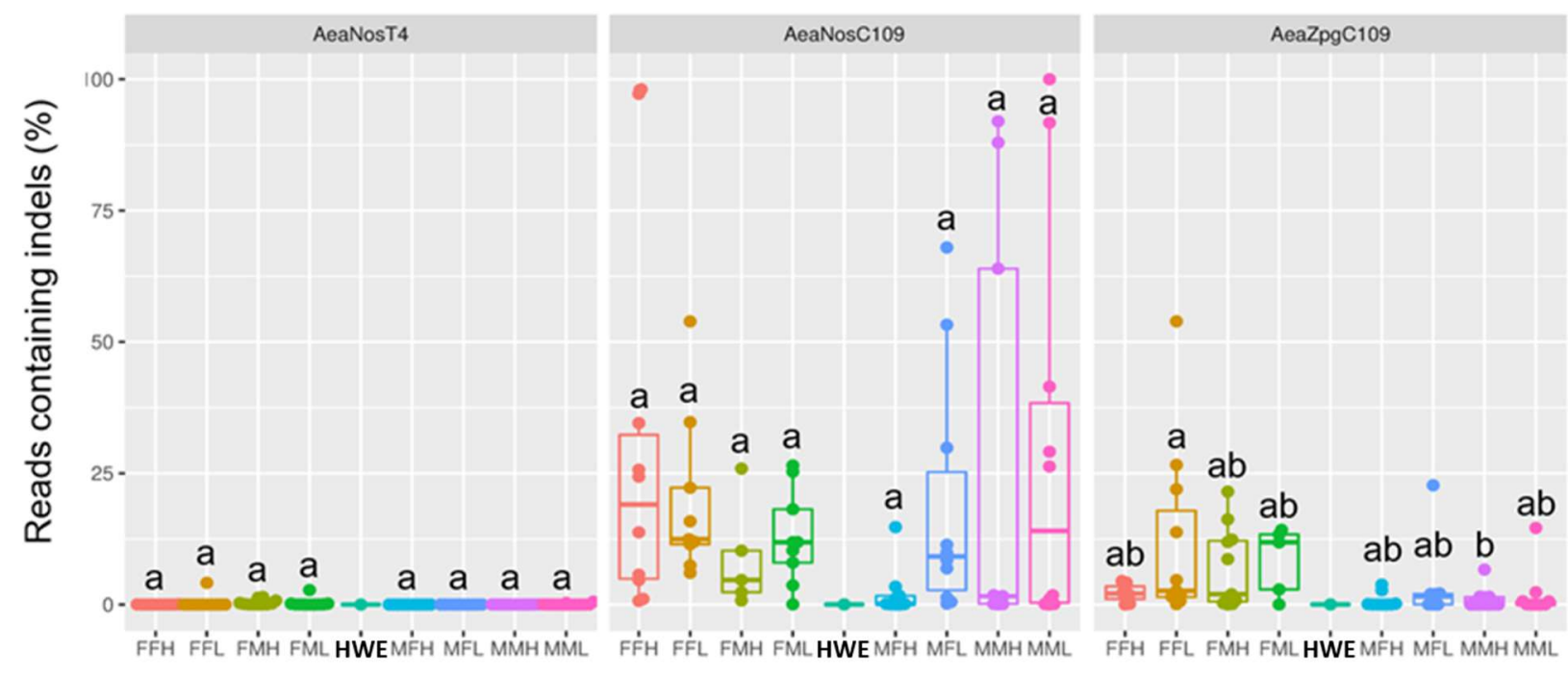

Parental/Grandparental transgenic sex for the outcross

Figure 3. Proportions of PCR amplicons containing GDBI for pooled non-transgenic mosquitoes from the second-outcrossed (OX-2) GD lines originating from either 'high' or 'low' parental drive pools with GD insertions at two different genomic loci (Carb109 or TIMP-P4) and Cas9 expression being controlled by two different promoters (nanos or $z p g$ ). The bar internal to the box represents the median value, while the lower and upper borders of the boxes represent Q1 and Q3, respectively. Indels were analyzed via high-throughput nucleotide sequencing. The first letter within each cross indicates the parental transgenic (OX-2) sex, the second letter indicates the grandparental (OX-1) transgenic sex. L=Low drive level in the parental generation, $\mathrm{H}=\mathrm{High}$ drive in the parental generation. $\mathrm{HWE}=\mathrm{Higgs}$ ' white eye wildtype. Each data point represents the percentage of high throughput reads containing a GDBI for PCR products that spanned the genomic target site for either Carb109 or TIMP-P4 locus. The gDNA template used for each PCR consisted of the pooled contribution of all non-transgenic larvae from the OX-2 crosses.

\section{Maternal and paternal contributions of the gene drive}

A major concern to an autonomous CRISPR/Cas9 gene drive system is that there are little-to-no maternal or paternal effects, which could result in cleavage of a wildtype allele in the absence of the gene drive cargo serving as the HDR donor. If the Cas9 ribonucleoprotein (Cas9-RNP) is available outside of the germline, as would be expected for a maternal/paternal contribution of Cas9-RNP, NHEJ is a favored mechanism of double-stranded DNA repair, which then leads to GDBI formation. To test for parental contribution in a locus in absence of a visible marker (such as white), we generated another transgenic line, AeaeCFPC109, containing a 3xP3-eCFP-SV40 marker at the identical Carb109 locus as the mCherry-marked AeaNosCarb109 ${ }^{\mathrm{GD}}$ line (Figure 4A). 
A)

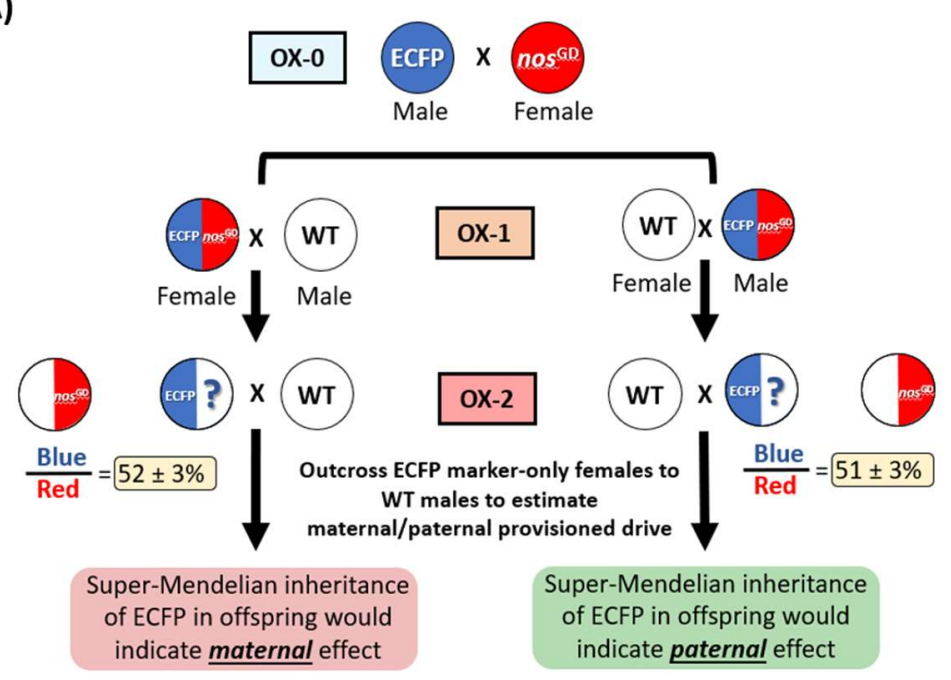

B) $100-$

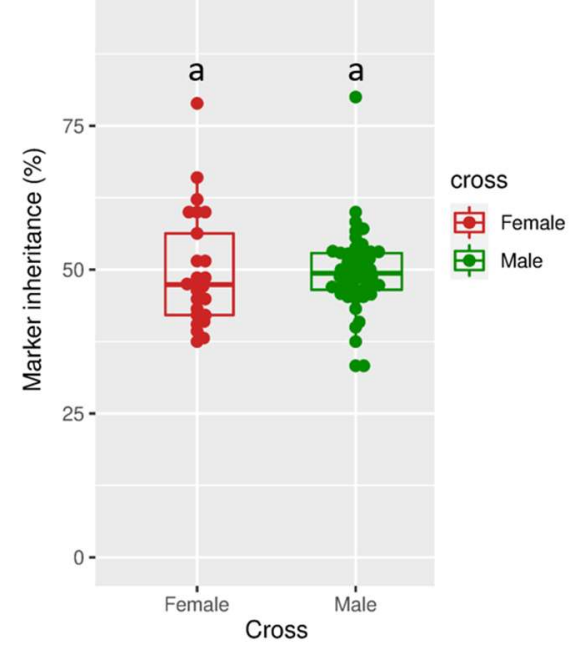

Figure 4. Locus balancing of an eCFP marker against the mCherry-marked AeaNosCarb109 ${ }^{\mathrm{GD}}$ line to test for parental drive effects. A) Crossing schematic for establishing trans-hemizygous parentals (OX-1), which were then outcrossed to wildtype HWE mosquitoes to obtain the eCFP parentals for OX-2. The eCFP parentals used for OX-2 contained the eCFP marker in the Carb109 locus, allowing for drive into a wildtype allele if trans-acting Cas9-RNP was provisioned either maternally or paternally. B) eCFP marker inheritance for the final outcrossing of OX-2 to wildtype, where inheritance $>50 \%$ is suggestive of parentally supplied Cas9-RNP. Each data point in (B) represents the percentage of transgene inheritance resulting from the offspring of the second outcrossing of eCFP positive to wildtype HWE males. The bar internal to the box represents the median value, while the lower and upper borders of the boxes represent Q1 and Q3, respectively. Groups superseded by the same letter are not significantly different $(p>0.05)$. A minimum of 20 larvae was set for each group in order to be scored.

By crossing these lines to obtain trans-hemizygous mosquitoes, the gene drive was blocked by the eCFP marker which could serve as a donor for gene drive into a wildtype allele if active Cas9-RNP targeting the Carb109 locus was provisioned maternally/paternally. In addition, the contributed wildtype allele could be targeted for NHEJ by any parentally supplied Cas9-RNP in the early embryo. In the first outcross (OX-1) of the trans-hemizygous individuals, we found that the inheritance of the blue eCFP marker and the mCherry-marked gene drive was nearly 50\% (Figure 4B) for both females and males and was not statistically different when tested using pairwise T-testing ( $p=0.5911$ and $p=0.7587$, respectively). This result suggested that there was no difference in fitness cost at the gamete level between an eCFP marker and the gene drive construct at the Carb109 locus. When the eCFP-inheriting individuals were further outcrossed to wildtype (OX-2) to investigate the level of parental gene drive contribution, the median level of inheritance was $47 \%$ for offspring from eCFP females and $49 \%$ for offspring with eCFP male parentals; however, some data points were greater than $50 \%$ with two points reaching $80 \%$

(Figure 4B). These results suggested that parental contribution of Cas9-RNP did not result in gene drive, 
but it was still possible that a parental effect could be contributing to the formation of GDBI. Therefore, we assessed up to five eCFP-negative larvae from each of the crosses and conducted PCR and Sanger sequencing across the Carb109 locus to determine if indel formation had occurred at the alternately inherited null allele from the eCFP parentals. Overall, a total of 293 non-transgenic larvae were analyzed for parental contribution of GDBI. Of these larvae, 118 descended from 24 female eCFP parentals that originated from eight outcrosses (OX-2) of female trans-hemizygous mosquitoes to wildtype HWE males. The remaining 175 larvae descended from 35 female eCFP parentals that came from eight outcrosses (OX-2) of male trans-hemizygous mosquitoes to wildtype HWE females. Following PCR amplification across the Carb109 locus, none of the 293 mosquitoes sampled contained GDBI. These results suggested that there was no parental contribution of the Cas9-RNP complex that carried through to the early embryo.

\section{Discussion}

The spatio-temporal expression of transgenes in Ae. aegypti is strongly influenced by the promoter used for expression as well as the genomic transgene insertion site, which is affected by position effect variegation (Franz et al., 2009; Henikoff,1992). The importance of genomic position for optimal Cas9 activity in Ae. aegypti was identified in the work by Li et al. (2017), where the activity of Cas 9 expression varied by genomic locus when placed under control of six different promoters and integrated into the Ae. aegypti genome in a quasi-random fashion using the piggyBac TE. The forward approach from Li et al. (2017) allowed for the identification of optimal genomic insertion loci for germline Cas9 activity, while in our study, we took a reverse approach by utilizing two genomic loci (TIMP-P4 and Carb109) previously identified to be permissive to antiviral transgene expression. Our reasoning for this was that as long as the gene drive cassettes were positioned in a genomic locus that supported the strong expression of antiviral effectors, the entire system could be built and established as a single-component autonomous gene drive line. The maintenance of a single locus in such a genetically modified mosquito line would simplify the future development of a gene drive line for a population replacement approach since only a single locus would need to be monitored and driven through the population. Our reverse engineering approach demonstrated that genomic loci that are well-suited towards the expression of transgenes upon blood-feeding may not necessarily be ideal for the functioning of a CRISPR/Cas9 autonomous gene drive system. Notably, the presence of the gene drive system at the TIMP-P4 locus resulted in no gene drive activity and further demonstrated a very low level of inheritable GDBI when assessed using whole-amplicon sequencing. By contrast, the TIMP-P4 locus exhibited strong transgene 
expression upon blood-feeding when they were placed under control of the carboxypeptidase $A$ promoter (AAEL010782) (Dong et al., 2014; Williams et al., 2020). Meanwhile, a second genomic locus that had also been shown to be ideal for stable antiviral activity (i.e.: Carb109; Franz et al., 2014) was highly permissive for gene drive activity, with some crosses reaching $100 \%$ inheritance. However, while the Carb109 locus allowed for strong levels of autonomous gene drive, there was a concomitant level of GDBI detectable that arose within the various crosses. This demonstrated that while the Carb109 locus supported gene drive activity, it resulted in heritable gene drive resistant alleles, which could ultimately block the gene drive in a population. Taken together, these results demonstrated that an autonomous gene drive system for the purposes of a population replacement strategy to drive an antiviral effector gene through a population requires additional surveying of genomic loci to optimize for both the gene drive system and the antiviral effector, and that our forward approach to identify suitable integration loci is feasible. The original autonomous drive in An. stephensi placed the expression of Cas9 under control of the vasa promoter and targeted the kh gene, which allowed for tracking of the autonomous drive system through the DsRed eye marker, as well as through $k h^{-} / k h^{-}$white eye phenotype for both somatic and inheritable GDBI (Gantz et al., 2015). The authors showed that the $k h$ locus allowed for both high levels of gene drive as well as blood meal-inducible expression of two anti-Plasmodium effector genes. Further, it was observed that while drive levels were highly efficient, GDBI were detectable and inheritable. There was also an associated negative selection for $k h^{-} / k h^{-}$mutants. Eventually, the gene drive was further modified by Adolfi et al. (2020) to provide an in-frame recoded rescue of the $k h$ gene, which resulted in an autonomous drive that spread through a population within as few as five generations because the inheritance of the drive system also conferred a rescue version of the $k h$ allele. In our autonomous drive at the Carb109 locus, the insertion target is intergenic, and as such, no associated negative selection would be expected. This is most likely the explanation for the high level of GDBI observed for the AeaNosC109GD line and for female parentals of the AeaZpgC109 ${ }^{G D}$ line since there would be no expected negative selection for NHEJ events at the target locus. Gantz et al. (2015) further predicted that there was early embryonic activity of Cas9-RNP prior to soma/germline differentiation, owing to the maternally provisioned activity of the vasa promoter. For this reason, we included two promoters previously demonstrated to be restricted to the germline: the nanos promoter in Ae. aegypti (Adelman et al., 2007) and the Ae. aegypti zpg promoter, previously reported in An. gambiae (Hammond et al., 2016; Kyrou et al., 2018). Carballar-Lejarazú et al. (2020) recently utilized the nanos promoter to establish an autonomous gene drive system at the cardinal gene locus in An. gambiae and conducted cage trials to demonstrate that a 1:9 (transgenic : wildtype) release ratio would result in full penetrance 
of the drive into the population within six generations with negligible $(<1 \%)$ gene drive-blocking indel accumulation. In comparison, when the AeaNosC109 ${ }^{\mathrm{GD}}$ line was assessed for inheritable GDBI for the OX-1 cross, we revealed that while the drive reached $89 \%$ inheritance, more than half $(7 / 12)$ of the mosquitoes that did not inherit the gene drive contained GDBI. Moreover, GDBI were identified in progeny that came from both male and female transgenic parentals. In the autonomous gene drive line developed by Carballar-Lejarazú et al. (2020), the cardinal gene target is the final step in the ommochrome (eye pigment) biosynthesis pathway. Unlike the $k h^{-}$mutants in An. stephensi, the eye pigmentation in cardinal individuals will gradually oxidize into the final native state and consequently, no associated fitness cost was observed in the AgNosCd1 ${ }^{\mathrm{GD}}$ line (Carballar-Lejarazú et al., 2020). In our study, we tested genomic loci that would not result in a phenotypic difference if GDBI were present. Therefore, to compare our autonomous gene drive system to the split drive system from Li et al. (2020) and Verkuijl et al. (2020), we used a drive-blocking eCFP marker at the Carb109 locus to assess maternal or paternal contribution of the Cas9-RNP in the early embryo, which allowed us to perform reciprocal trans-heterozygote crosses to wildtype as outlined in Li et al. (2020). Outcrossing of the eCFP-positive mosquitoes showed no increase in inheritance, and furthermore, no GDBI were identified. Taken together these results suggest that there is no maternal or paternal carryover of Cas9-RNP when Cas9 is placed under control of the nanos promoter and the sgRNA is expressed from the U6:3 promoter AAEL017774. Since the Cas9 is under control of the maternal nanos gene promoter, it would be expected that Cas9 mRNA would be provided maternally. An elegant study by Terradas et al (2021) demonstrated that the nanos-driven Cas9 transcripts from the $\mathrm{AgNosCd} 1^{\mathrm{GD}}$ line (Carballar-Lejarazú et al., 2020) were indeed expressed in the nurse cells along with native nanos transcripts; however, unlike the native nanos transcripts, the Cas9 transcripts were found only at very low levels or were absent in the egg. By contrast, in males, Cas9 expression driven by the nanos regulatory elements paralleled that of the native nanos transcript. This then could explain the observed activity of gene drive for male parentals in the AegNosC109 ${ }^{G D}$ line and the absence of a maternal effect. Further, Terradas et al (2021) showed that in An. stephensi, while $\beta 2$-tubulin transcripts were male-specific, they were also expressed in the late stages of spermatogenesis, which would preclude the use of the $\beta 2$-tubulin for the purposes of a CRISPR/Cas9 based gene drive. Likely, this was the cause of the absence of gene drive that we observed for the Aeaß2tC109 ${ }^{\mathrm{GD}}$ line. In addition, Terradas et al (2021) investigated the pattern of native zpg expression and found that the expression was similar to vasa but at a lower expression level. They found that there was expression of $z p g$ throughout premeiotic and meiotic stages in males and highlevel zpg expression in the sperm flagella, which could increase the prevalence of GDBI. In our 
AeaZpgC109GD line, however, we identified a lower level of GDBI for male parentals and a lower level of gene drive compared to the AeaNosC $109^{G D}$ line. Thus, it is likely that the expression of Cas 9 transcript under control of the zpg promoter does not perfectly parallel that of the native zpg transcript. As a consequence, the absence of a maternal effect causing Cas9 activity was either due to the ideal localization of Cas9 to the posterior end of the early embryo, as demonstrated for native Ae. aegypti nanos mRNA (Calvo et al., 2005), or due to insufficient/no sgRNA contribution. These results strongly support the combined usage of the nanos promoter for the expression of the Cas9 and the AAEL017774 promoter for the expression of the sgRNA in an autonomous gene drive system in Ae. aegypti. An ideal target might be the cardinal gene identified in An. gambiae, which resulted in negligible GDBI and reached fixation in cage populations within five generations (Carballar-Lejarazú et al. 2020), or the $k h$ gene, which was shown to support both gene drive activity and the expression of anti-Plasmodium effectors (Gantz et al., 2015; Adolfi et al., 2020). While previous studies investigating gene drive in mosquitoes provided potential targets for autonomous gene drives in Ae. aegypti, they all focused on genes that resulted in an eye marker phenotype such as white, kynurenine hydroxylase, or cardinal (Li et al., 2020; Verkuijl et al., 2020; Gantz et al., 2015; Adolfi et al., 2020; Carballar-Lejarazú et al., 2020). By targeting genes that confer a visible phenotype, the levels of mosaicism as well as GDBI can be identified visually. While this simplifies the testing of the gene drives, it restricts the number of available genomic loci that can be assessed for their capacity to have both high gene drive activity/fidelity and high antipathogen expression/activity. Since we investigated intergenic loci in our study, we needed a method to assess for the allele alternate of the gene drive, which would allow us to test for the contribution of maternal and/or paternal contribution of the Cas9/sgRNA RNP complex. Through the construction of an alternate fluorescent eye marker positioned at the same CRISPR/Cas9 target site, we were able to balance the locus and block the gene drive such that an outcrossing of the double (trans)heterozygous mosquitoes would allow for the assessment of any CRISPR/Cas9 RNP activity in the form of either increased inheritance of the alternate fluorescent eye marker (gene drive) or the formation of GDBI. This approach allows for the testing of parental contribution and is permissive for the targeting of any genomic locus, independent of whether or not disruption of the locus results in a phenotypic change. Should it prove difficult to identify a single genomic locus that allows for a one-component autonomous gene drive that strongly expresses an antipathogen transgene, a multiple locus approach could be considered. Gene drive systems that would accommodate such a scenario, i.e., transcomplementary drives (Bier et al., 2019) and multiple loci drives (Yan and Finnegan, 2018), have been proposed. These gene drive systems would allow for the positioning of the antipathogen effector in the 
Carb109 or the TIMP-P4 locus, while placing the Cas9 driver in a region that restricts maternal and paternal effects and the formation of GDBI, such as exon III of the cardinal gene (Carballar-Lejarazú et al., 2020). Given that only a single polymorphism was observed for the Carb109 target site, and that the polymorphism was present at a low allelic frequency at the distal end of the protospacer, the Carb109 locus looks highly suitable as a noncoding region interrupting target for the driving of antipathogen effectors through Ae. aegypti populations.

Acknowledgements We would like to thank Drs. Hanno Schmidt and Gregory Lanzaro for kindly sharing the genomic vcf data for the Ae. aeygpti genomes. We also thank the University of Missouri Genomics Technology Core for technical support. Finally, we thank the NIH-NIAID for the funding for this project (R01 Al130085-01A1). Contributions: AWEF, KEO conceived the study, WR, AEW, RJ cloned constructs, WR, JL established the transgenic lines and performed crosses. WR, AEW, KEO, and AWEF wrote the paper.

\section{References}

Adelman ZN, Jasinskiene N, Onal S, Juhn J, Ashikyan A, Salampessy M, MacCauley T, James AA. 2007. Nanos gene control DNA mediates developmentally regulated transposition in the yellow fever mosquito Aedes aegypti. Proc Natl Acad Sci USA. 104(24):9970-9975. DOI:10.1073/pnas.0701515104.

Adolfi A, Gantz VM, Jasinskiene N, Lee H-F, Hwang K, Terradas G, Bulger EA, Ramaiah A, Bennett JB, Emerson JJ, Marshall JM, Bier E, James AA. 2020. Efficient population modification gene-drive rescue system in the malaria mosquito Anopheles stephensi. Nat Commun 11, 5553. DOI:10.1038/s41467-02019426-0.

Anderson MA, Gross TL, Myles KM, Adelman ZN. 2010. Validation of novel promoter sequences derived from two endogenous ubiquitin genes in transgenic Aedes aegypti. Insect Mol Biol. 19(4):441-449. DOI:10.1111/j.1365-2583.2010.01005.x.

Basu S, Aryan S, Overcash JM, Samuel GH, Anderson MAE, Dahlem TJ, Myles KM, Adelman ZN. 2015. CRISPR-based gene insertion in mosquitoes. Proc Natl Acad Sci USA. 112(13):4038-4043. DOI:

10.1073/pnas.1502370112.

Beebe NW, Pagendam D, Trewin BJ, Boomer A, Bradford M, Ford A, Liddington C, Bondarenco A, De Barro PJ, Gilchrist J, Paton C, Staunton KM, Johnson B, Maynard AJ, Devine GJ, Hugo LE, Rasic G, Cook H, Massaro P, Snoad N, Crawford JE, White BJ, Xi Z, Ritchie SA. 2021. Releasing incompatible males drives strong suppression across populations of wild and Wolbachia -carrying Aedes aegypti in Australia. Proc Natl Acad Sci USA. 2021 Oct 12; Vol. 118 (41).

Bier E, Harrison MM, O'Connor-Giles KM, Wildonger J. 2018. Advances in Engineering the Fly Genome with the CRISPR-Cas System. Genetics. 208:1-18. DOI:10.1534/genetics.117.1113. 
Buchman A, Gamez S, Li M, Antoshechkin I, Li H-H, Wang H-W, Chen C-H, Klein MJ, Duchemin J-B, Paradkar PN, Akbari OS. 2019. Engineered resistance to Zika virus in transgenic Aedes aegypti expressing a polycistronic cluster of synthetic small RNAs. Proc. Natl. Acad. Sci. USA. 116(9):3656-3661. doi: 10.1073/pnas.1810771116.

Buchman A, Gamez S, Li M, Antoshechkin I, Li HH, Wang HW, Chen CH, Klein MJ, Duchemin JB, Crowe JE Jr, Paradkar PN, Akbari OS (2020) Broad dengue neutralization in mosquitoes expressing an engineered antibody. PLoS Pathog 16(1):e1008103.

Burt A. 2003. Site-specific selfish genes as tools for the control and genetic engineering of natural populations. Proc Biol Sci. 270(1518):921-928. doi:10.1098/rspb.2002.2319.

Calvo E, Walter M, Adelman ZN, Jimenez A, Onal S, Marinotti O, James AA. 2005. Nanos (nos) genes of the vector mosquitoes, Anopheles gambiae, Anopheles stephensi and Aedes aegypti. Insect Biochem. Mol. Biol. 35(7):789-798.

Carballar-Lejarazú R, Ogaugwu C, Tushar T, Kelsey A, Pham TB, Murphy J, Schmidt H, Lee Y, Lanzaro GC, James AA. 2020. Next-generation gene drive for population modification of the malaria vector mosquito, Anopheles gambiae. Proc Natl Acad Sci USA. 15;117(37):22805-22814. doi: 10.1073/pnas.2010214117.

Cencic R, Miura H, Malina A, Robert F, Ethier S, Schmeing TM, Dostie J, Pelletier J. 2014. Protospacer adjacent motif (PAM)-distal sequences engage CRISPR Cas9 DNA target cleavage. PLoS One. 9(10):e109213. DOI:10.1371/journal.pone.0109213.

Certo MT, Ryu BY, Annis JE, Garibov M, Jarjour JV, Rawlings DJ, Scharenberg AM. 2011. Tracking genome engineering outcome at individual DNA breakpoints. Nat Methods. 2011;8(8):671-676.

DOI:10.1038/nmeth.1648.

Chae, K., C. Dawson, C. Valentin, K. Myles, and Z. Adelman. 2020. Engineering a biodegradable transgene in the dengue mosquito Aedes aegypti. 2020 Entomology Virtual Annual Meeting. Entomological Society of America. http://www.entsoc.org/events/annual-meeting.

Clement K, Rees H, Canver MC, Gehrke JM, Farouni R, Hsu JY, Cole MA, Liu DR, Joung K, Bauer DE, Pinello L. 2019. CRISPResso2 provides accurate and rapid genome editing sequence analysis. Nat Biotechnol 37, 224-226. DOI:10.1038/s41587-019-0032-3.

Dang Y, Jia G, Choi J, Ma H, Anaya E, Ye C, Shankar P, Wu H. 2015. Optimizing sgRNA structure to improve CRISPR-Cas9 knockout efficiency. Genome Biol 16, 280 DOI:10.1186/s13059-015-0846-3.

Dong S, Balaraman V, Kantor AM, Lin J, Grant DG, Held NL, Franz AWE. 2017. Chikungunya virus dissemination from the midgut of Aedes aegypti is associated with temporal basal lamina degradation during bloodmeal digestion. PLOS Negl. Trop. Dis. 11(9):e0005976. doi: 10.1371/journal.pntd.0005976

Du Y, Nomura Y, Zhorov B, Dong K. 2016. Sodium channel mutations and pyrethroid resistance in Aedes aegypti. Insects 7:60.

Engler C, Kandzia R, Marillonnet S. 2008. A One Pot, One Step, Precision Cloning Method with High Throughput Capability. PLoS ONE 3(11):e3647. DOI:10.1371/journal.pone.0003647.Fan Y, Scott JG. 2020. 
The F1534C voltage-sensitive sodium channel mutation confers 7- to 16-fold resistance to pyrethroid insecticides in Aedes aegypti. Pest Manag Sci. 76(6):2251-2259. doi: 10.1002/ps.5763.

Florida Keys Mosquito Control District. 2019. How we control mosquitoes. http://keysmosquito.org/control-methods/\#1485280164900-759a53c4-6cb7.

Franz AW, Sanchez-Vargas I, Piper J, Smith MR, Khoo CC, James AA, Olson KE. Stability and loss of a virus resistance phenotype over time in transgenic mosquitoes harbouring an antiviral effector gene. Insect Mol Biol. 2009 Oct;18(5):661-72. doi: 10.1111/j.1365-2583.2009.00908.x. PMID: 19754743; PMCID: PMC4839482.

Franz AWE, Sanchez-Vargas I, Raban RR, Black WC IV, James AA, Olson KE. 2014. Fitness Impact and Stability of a Transgene Conferring Resistance to Dengue-2 Virus following Introgression into a Genetically Diverse Aedes aegypti Strain. PLoS Negl Trop Dis 8(5): e2833. DOI:10.1371/journal.pntd.0002833.

Franz AW, Sanchez-Vargas I, Adelman ZN, Blair CD, Beaty BJ, James AA, Olson KE. 2006. Engineering RNA interference-based resistance to dengue virus type 2 in genetically modified Aedes aegypti. Proc. Natl. Acad. Sci. USA. 103(11):4198-203. doi: 10.1073/pnas.0600479103.

Gantz VM, Bier E. 2015. The mutagenic chain reaction: A method for converting heterozygous to homozygous mutations. Science 348(6233):442-444. doi:10.1126/science.aaa5945.

Gantz, VM, Bier E. 2016. The dawn of active genetics. Bioessays 38: 50-63.

Gantz VM, Jasinskiene N, Tatarenkova O, Fazekas A, Macias VM, Bier E, James AA. 2015. Cas9-mediated gene drive in Anopheles stephensi. Proc. Natl. Acad. Sci. USA 112(49):E6736-E6743. doi:10.1073/pnas.1521077112.

Gokcezade J, Sienski G, Duchek P. 2014. Efficient CRISPR/Cas9 plasmids for rapid and versatile genome editing in Drosophila. G3 (Bethesda). 17;4(11):2279-82. DOI: 10.1534/g3.114.014126.

Hammond A, Galizi R, Kyrou K, Simoni A, Siniscalchi C, Katsanos D, Gribble M, Baker D, Marois E, Russell S, Burt A, Windbichler N, Crisanti A, Nolan T. 2016. A CRISPR-Cas9 gene drive system targeting female reproduction in the malaria mosquito vector Anopheles gambiae. Nat. Biotechnol. 34(1):78-83. doi:10.1038/nbt.3439.

Henikoff S. 1992. Position effect and related phenomena. Curr. Opin. Genet. Devel. 2(6):907-912.

Horn C, Jaunich B, Wimmer EA. 2000. Highly sensitive, fluorescent transformation marker for Drosophila transgenesis. Dev. Genes Evol. 210(12): 623--629.

Irvin N, Hoddle MS, O'Brochta DA, Carey B, Atkinson PW. 2004. Assessing fitness costs for transgenic Aedes aegypti expressing the GFP marker and transposase genes. Proc. Natl. Acad. Sci. USA. 101(3):891896. doi:10.1073/pnas.0305511101.

Kittayapong P, Ninphanomchai S, Limohpasmanee W, Chansang C, Chansang U, Mongkalangoon P. 2019. Combined sterile insect technique and incompatible insect technique: The first proof-of-concept to 
suppress Aedes aegypti vector populations in semi-rural settings in Thailand. PLoS Negl Trop Dis. 13(10):e0007771. doi: 10.1371/journal.pntd.0007771.

Konet DS, Anderson J, Piper J, Akkina R, Suchman E, Carlson J. 2007. Short-hairpin RNA expressed from polymerase III promoters mediates RNA interference in mosquito cells. Insect Mol Biol. 16(2):199-206. DOI: $10.1111 /$ j.1365-2583.2006.00714.x.

Kyrou K, Hammond AM, Galizi R, Kranjc N, Burt A, Beaghton AK, Nolan T, Cristani A. 2018. A CRISPRCas9 gene drive targeting doublesex causes complete population suppression in caged Anopheles gambiae mosquitoes. Nat. Biotech.36:1062-1066.

Labbé GMC, Scaife S, Morgan SA, Curtis ZH, Alphey L. 2012 Female-Specific Flightless (fsRIDL) Phenotype for Control of Aedes albopictus. PLOS Neglected Tropical Diseases 6(7): e1724. doi.org/10.1371/journal.pntd.0001724.

Labun K, Montague TG, Gagnon JA, Thyme SB, Valen E.2016. CHOPCHOP v2: a web tool for the next generation of CRISPR genome engineering. Nucleic Acids Res. 8;44(W1):W272-6. DOI:

10.1093/nar/gkw398.

Li M, Bui M, Yang T, Bowman CS, White BJ, Akbari OS. 2017. Germline Cas9 expression yields highly efficient genome engineering in a major worldwide disease vector, Aedes aegypti. Proc. Natl. Acad. Sci. USA. 114(49):E10540-E10549. doi:10.1073/pnas.1711538114.

Li M, Yang T, Bui M, Gamez S, Wise T, Kandul NP, Liu J, Alcantara L, Lee H, Edula JR, Raban R, Zhan Y, Wang Y, DeBeaubien N, Chen J, Sánchez C HM, Bennett JB, Antoshechkin I, Montell C, Marshall JM, Akbari OS. 2021. Suppressing mosquito populations with precision guided sterile males. Nat Commun. 12:5374. doi.org/10.1038/s41467-021-25421-w.

Li M, Yang T, Kandul NP, Bui M, Gamez S, Raban R, Bennett J, Sánchez C HM, Lanzaro GC, Schmidt H, Lee $\mathrm{Y}$, Marshall JM, Akbari OS. 2020. Development of a confinable gene drive system in the human disease vector Aedes aegypti. eLife 9, e51701.

Liu N. 2015. Insecticide Resistance in Mosquitoes: Impact, Mechanisms, and Research Directions. Annu. Rev. Entomol. 60:537-559.

Liu-Helmersson J, Brännström Å, Sewe MO, Semenza JC, Rocklöv J. 2019. Estimating Past, Present, and 700 Future Trends in the Global Distribution and Abundance of the Arbovirus Vector Aedes aegypti Under Climate Change Scenarios. Front Public Health 7:148.

Manjarres-Suarez A, Olivero-Verbel J. 2013. Chemical control of Aedes aegypti: a historical perspective. Rev. costarric. salud pública [online]. 2013, vol.22, n.1 68-75. Available from:

$<$ http://www.scielo.sa.cr/scielo.php?script=sci_arttext\&pid=S1409-

$14292013000100012 \&$ Ing=en\&nrm=iso>. ISSN 1409-1429.

Martin M. 2011. Cutadapt removes adapter sequences from high-throughput sequencing reads. EMBnet.journal, 17(1):10-12. DOI:10.14806/ej.17.1.200. 
Martins AJ, Lins RM, Linss JG, Peixoto AA, Valle D. 2009. Voltage-gated sodium channel polymorphism and metabolic resistance in pyrethroid-resistant Aedes aegypti from Brazil. Am J Trop Med Hyg. 81(1):108-15. PMID: 19556575.

Moreira LA, Wang J, Collins FH, Jacobs-Lorena M. 2004. Fitness of anopheline mosquitoes expressing transgenes that inhibit Plasmodium development. Genetics. 166(3):1337-41. doi:

10.1534/genetics.166.3.1337.

Saavedra-Rodriguez K, Maloof FV, Campbell CL, Garcia-Rejon J, Lenhart A, Penilla P, Rodriguez A, Sandoval AA, Flores AE, Ponce G, Lozano S, Black WC 4th. 2018. Parallel evolution of vgsc mutations at domains IS6, IIS6 and IIIS6 in pyrethroid resistant Aedes aegypti from Mexico. Sci Rep 8:6747.

Schluep SM, Buckner EA. 2021. Metabolic Resistance in Permethrin-Resistant Florida Aedes aegypti (Diptera: Culicidae). Insects 12:866. doi.org/10.3390/insects12100866.

Schmidt H, Collier TC, Hanemaaijer MJ, Houston PD, Lee Y, Lanzaro GC. 2020. Abundance of conserved CRISPR-Cas9 target sites within the highly polymorphic genomes of Anopheles and Aedes mosquitoes. Nat Commun 11, 1425. DOI:10.1038/s41467-020-15204-0.

Tazuke SI, Schulz C, Gilboa L, Fogarty M, Mahowald AP, Guichet A, Ephrussi A, Wood CG, Lehmann R, Fuller MT.2002. A germline-specific gap junction protein required for survival of differentiating early germ cells. Development. 129(10):2529-39.

Terradas G, Hermann A, James AA, McGinnis W, Bier E. 2021. High-resolution in situ analysis of Cas9 germline transcript distributions in gene-drive Anopheles mosquitoes, G3.

DOI:10.1093/g3journal/jkab369.

Verkuijl SAN, Gonzalez E, Li M, Ang J, Kandul NP, Anderson MAE, Akbari OS, Bonsall MB, Alphey L. 2020. A CRISPR endonuclease gene drive reveals two distinct mechanisms of inheritance bias. bioRxiv 2020.12.15.421271. doi: 10.1101/2020.12.15.421271.

Waltz E. 2021. First genetically modified mosquitoes released in the United States. Nature 593: 175-176.

Weaver SC, Reisen WK. 2010. Present and future arboviral threats. Antiviral Res. 85(2):328-45. doi: 10.1016/j.antiviral.2009.10.008.

Weaver SC, Vasilakis N. 2009. Molecular evolution of dengue viruses: contributions of phylogenetics to understanding the history and epidemiology of the preeminent arboviral disease. Infect Genet Evol. 9(4):523-40. doi: 10.1016/j.meegid.2009.02.003.

Williams AE, Sanchez-Vargas I, Reid WR, Lin J, Franz AWE, Olson KE. The Antiviral Small-Interfering RNA Pathway Induces Zika Virus Resistance in Transgenic Aedes aegypti. Viruses. 2020 Oct 30;12(11):1231. doi: $10.3390 / v 12111231$.

Windbichler N, Papathanos PA, Catteruccia F, Ranson H, Burt A, Crisanti A. 2007. Homing endonuclease mediated gene targeting in Anopheles gambiae cells and embryos. Nucleic Acids Res. 35(17):5922-5933. doi:10.1093/nar/gkm632.Wu B, Luo L, Gao XJ. 2016. Cas9-triggered chain ablation of Cas9 as a gene drive brake. Nat. Biotechnol. 34: 137-138. 
Wu, B., L. Luo, and X. J. Gao. 2016. Cas9-triggered chain ablation of cas9 as a gene drive brake. Nat. Biotechnol. 34: 137-138.

Yan Y, Finnigan GC. 2018. Development of a multi-locus CRISPR gene drive system in budding yeast. Sci Rep 8:17277. DOI:10.1038/s41598-018-34909-3.

Yen PS, James AA, Li JC, Chen CH, Failloux AB (2018) Synthetic miRNAs induce dual arboviral-resistance phenotypes in the vector mosquito Aedes aegypti. Commun Biol 1:11.

Zapletal J, Najmitabrizi N, Erraguntla M, Lawley MA, Myles KM, Adelman ZN. 2021. Making gene drive biodegradable. Phil. Trans. R. Soc. B 376: 20190804. DOI: 10.1098/rstb.2019.0804. 